\title{
Man-Machine Synergy Control for Pneumatically Powered Exoskeleton Based on Surface Electromyogram Signal
}

\author{
Feilong Jiang $\mathbb{D D}^{1}{ }^{1}$ Hao Liu, ${ }^{2}$ Qingwei Li, ${ }^{3}$ Jian Cao, ${ }^{1}$ Xiaoliang Yin, ${ }^{1}$ and Rui Dong ${ }^{1}$ \\ ${ }^{1}$ Jia Xing University, Jiaxing, China \\ ${ }^{2}$ State Key Laboratory of Fluid Power and Mechatronic Systems, Zhejiang University, Hangzhou, China \\ ${ }^{3}$ Yunnan University of Bussiness Management, Kunming, China \\ Correspondence should be addressed to Feilong Jiang; jiangfeilong2007@163.com
}

Received 10 December 2021; Revised 27 December 2021; Accepted 26 January 2022; Published 22 February 2022

Academic Editor: Xindong Peng

Copyright ( $\odot 2022$ Feilong Jiang et al. This is an open access article distributed under the Creative Commons Attribution License, which permits unrestricted use, distribution, and reproduction in any medium, provided the original work is properly cited.

\begin{abstract}
The biceps and triceps alternatively act as agonists and antagonists to realize upper limb movement. Pneumatic artificial muscle (PAM), which is inflated and deflated with compressed air instead of water, has similar characteristics to those of human muscle. The challenge is whether an exoskeleton actuated by PAM can help biceps lift the upper limb. Accordingly, the principal aim of this research is to guarantee precise signal collection and control process and adopt the synergy control of PAM and upper limb. In this system, the biceps and triceps provide the main signals in synergy control, electrodes are pasted outside of biceps and triceps to sample their electromyogram signal (EMGs), and the mechanical structure and control system of the pneumatic exoskeleton are proposed. The relationship between duty-ratio-controlled variables and PAM contraction speed is given by experimental analysis, and the maximum duty ratio of controlled variables of input is set to 80 . The feature analysis of EMGs can be various including envelope, moving average, and moving root mean square (RMS). The envelope is taken to extract muscle contraction information through upper limb muscles in a static contraction experiment. Then, the processes of biceps and triceps EMGs feature changes including rapid swing, slow swing, and discontinuous swing under various loads are analyzed during upper limb muscle dynamic contraction. The duty-ratio-controlled variables can be divided into five levels, which correspond to exertion rating from powerless to very strong in two EMG characters. These can be reflected in a scatter diagram of duty-ratio-controlled variables and average EMG characters. A nonlinear relationship can be transferred into the continuous system by the polynomial interpolation method, solving the problem of saturation. The net duty-ratio-controlled variables are adopted to control the on-off state and pulse-width modulation (PWM) duty ratio of the high-speed on-off valve. The forearm lifting up movement is unpowered and powered with various load EMGs, and elbow discontinuous swing angle overshoot is performed to analyze the coordination effect in a synergy control experiment.
\end{abstract}

\section{Introduction}

The exoskeleton has become one of the important research subjects in recent years, especially in gait training [1], rehabilitation [2,3], and tremor-suppression orthoses [4]. Almost all existing actuators adopt a driven exoskeleton, including a motor [5-9], hydraulic system [10], pneumatic system [11], and wires [12, 13]. Motors possess the advantages of small volume, low weight, large force of traction, simple operation, good performance, and high safety; therefore, they have been widely used in artificial exoskeletons. The relevant research work mainly focuses on the control algorithm to reduce the error; examples of solutions include adaptive neural network fast fractional sliding mode control [7] and standard force feedback control such as interaction force feedback control and the reference generation [8], adaptive impedance control [9], or sliding mode control [11].

The above researches mainly focus on the active rehabilitation of the exoskeleton; studies on load carriage represent another research direction. Nonetheless, electromyogram signals (EMGs), which reflect muscle and joint actual condition, have been rarely considered. For example, a motor combined with EMGs was presented for upper limb rehabilitation [14]. Muscles are often in more than one state at any given time; that is, they can be in 
isobaric, isotonic, and isometric contraction and expansion. Pneumatic artificial muscle (PAM), which is much lighter and, at the same time, more compliant than a traditional motor, has similar properties as human muscles; therefore, it is a better choice for movement rehabilitation.

This paper proposes an exoskeleton for load carriage actuated by PAM. Its mechanical structure, the control system of the pneumatic exoskeleton, and the relationship between duty-ratio-controlled variables and PAM contraction speed are presented in Section 2. Section 3 experimentally evaluates the upper limb muscles' static contraction characteristics. Section 4 illustrates the AR model process flow and upper limb muscles dynamic contraction characteristics. In Section 5, the experimental verification of the coordination effect in synergy control is presented.

\section{Mechanism and Control Principle}

As can be seen in Figure 1, the pneumatic exoskeleton chiefly consists of a posterior arm, a forearm, a baffle, an angular transducer, a plate, and PAM. The posterior arm is sufficiently tightened to hold the articulatio humeri in position, in order to provide a stable point of attachment of PAM that remains relatively fixed during contraction. Forearm lift-up motion occurs as a result of PAM contraction, where a cable pulls a pulley in the elbow, and the forearm revolves around the elbow. Baffles are mounted individually onto the posterior arm and forearm, and the upper limb is fixed onto baffles by the bandage. An angular transducer is set on the elbow, which measures angle changes in the forearm. The tester wears short-sleeved shirts, and the baffle is in direct contact with the arm in actual use. A dumbbell is used for additional load in the plate, which makes it more convenient to increase and decrease the applied load.

The control principle of the proposed pneumatic exoskeleton is shown in Figure 2, where the host computer outputs pulse-width modulation (PWM) signals to a data acquisition card, and then, a single-chip microcomputer performs further processing. After the single-chip microcomputer receives signals, it will allow for the corresponding PWM signals' duty cycle and frequency to be drawn out of the amplifier, which will, in turn, control the opening and closing of a high-speed on-off valve. The sampled data of angle change in the elbow flow through the signal adjust circuit, and then, it is transmitted to the data acquisition card and host computer. The host computer also shows the collection of EMGs by electrodes, which are attached outside of the biceps and triceps [15]. The PAM is in the condition of either being deflated or inflated according to collected data of EMGs and angle changes.

The MHE2-MS1H-3/2G-QS-4-K (FESTO company) is selected as a high-speed on-off valve. The most suitable valve is found by studying the relationship between the high-speed on-off valve duty cycle and PAM contraction speed.

With the plate carrying a $3 \mathrm{~kg}$ weight, the duty ratio of the high-speed on-off valve is simultaneously altered, the recording time is from $3^{\circ}$ to $60^{\circ}$ in the elbow, and movements can be roughly divided into two types: up and down. The

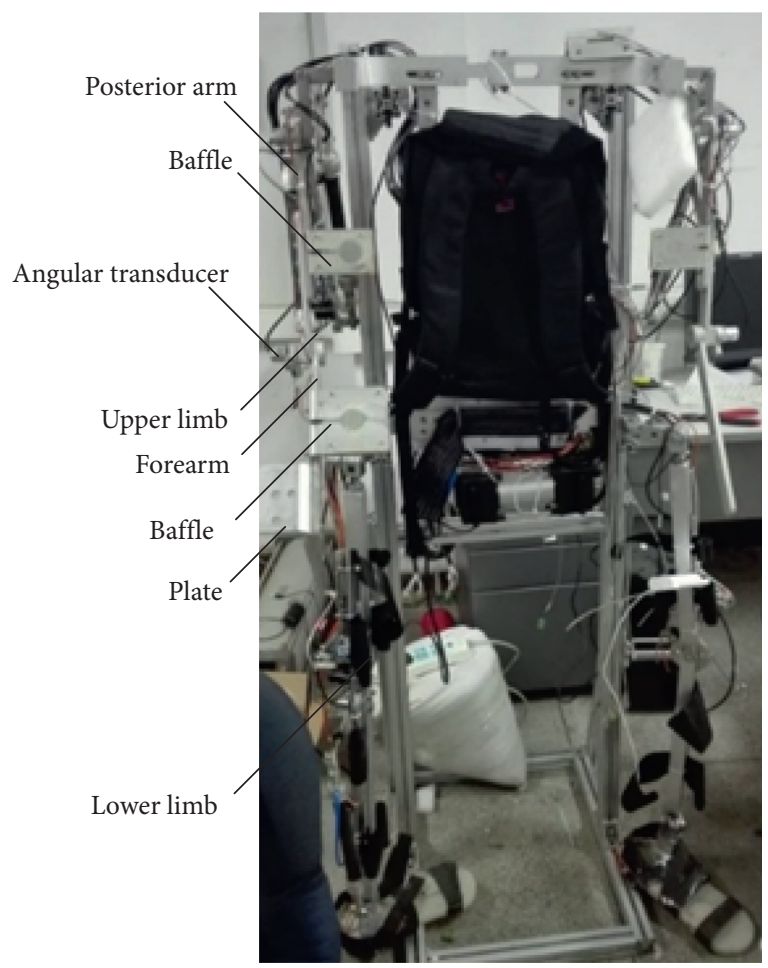

FIGURE 1: Overall mechanical structure of pneumatic exoskeleton.

relationship between duty-ratio-controlled variables and contraction speed is shown in Figure 3, which can evaluate the speed of the exoskeleton according to the inverse of elapsed time.

Figure 3 shows that when the value of duty-ratiocontrolled variables is less than 80 , it shows approximating linear relationships with the increase and decrease of speed. The elbow rotational speed reduces as the value of duty-ratio-controlled variables continues to increase. Moreover, it is experimentally found that the maximum permissible comfortable rotational speed in the elbow occurs when the duty-ratio-controlled variable is 80 . Thus, the maximum duty-ratio-controlled variable of input is set to 80 , and the problem of speed limit can be resolved, while the requirement of linear PAM contraction speed can be met.

\section{Static Contraction of Upper Limb Muscles}

3.1. Purpose and Experimental Design. With a constant elbow joint angle, we investigate how the EMGs of biceps and triceps change over time when different weights are loaded.

We try to imitate how the human limb works using the proposed exoskeleton, picking up and analyzing the EMGs when the exoskeleton is attached to the tester. Electrodes are laid outside of the biceps and triceps, and the posterior arm and forearm are fastened to the exoskeleton by the bandage. The posterior arm is in a vertical position, while the forearm is horizontal. Both upper limbs of the tester and the exoskeleton are lifted from the initial position to a set position, kept still for a period of 10 seconds, dropped, and returned to the initial position. 


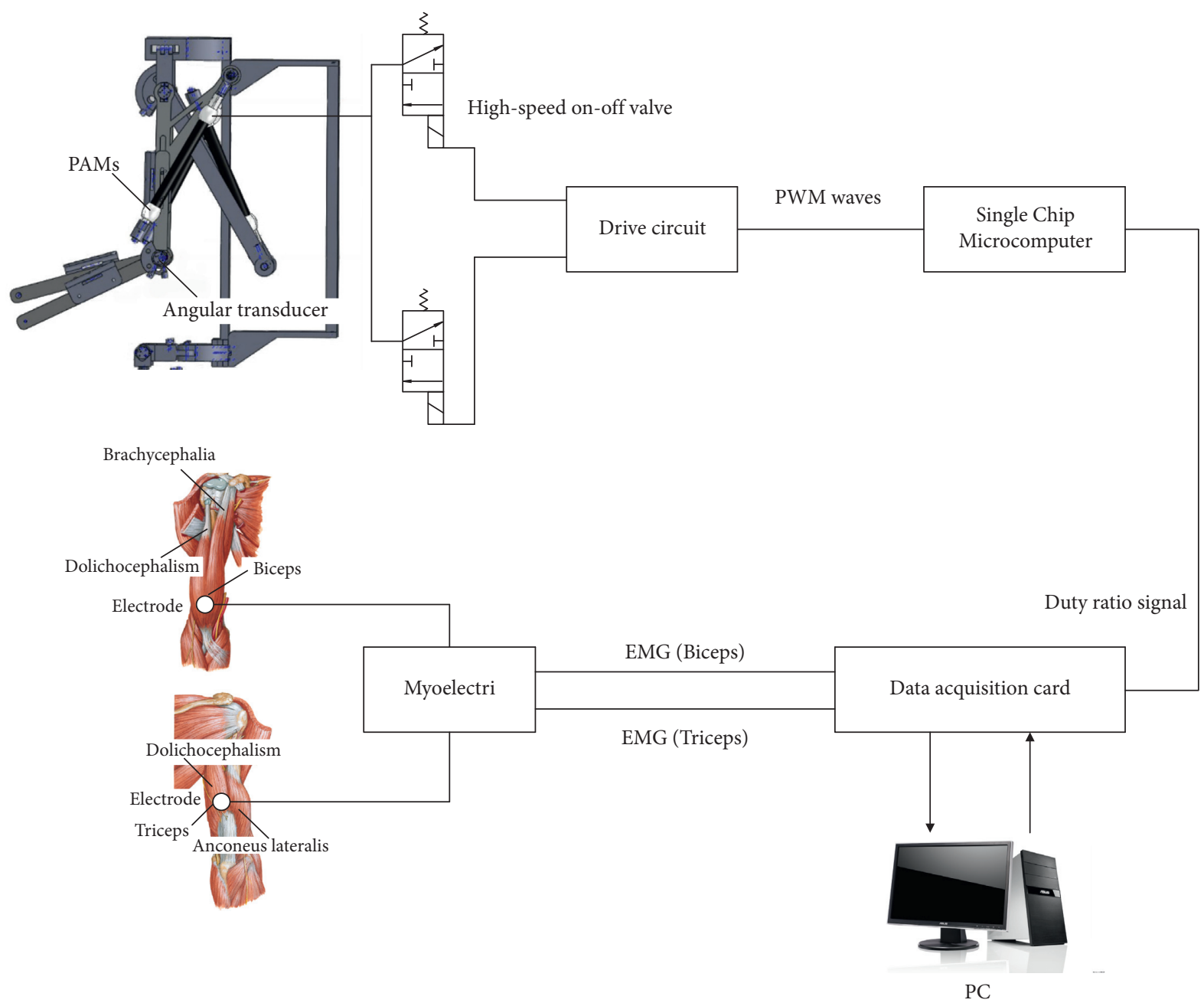

Figure 2: Control principle of the pneumatic exoskeleton.

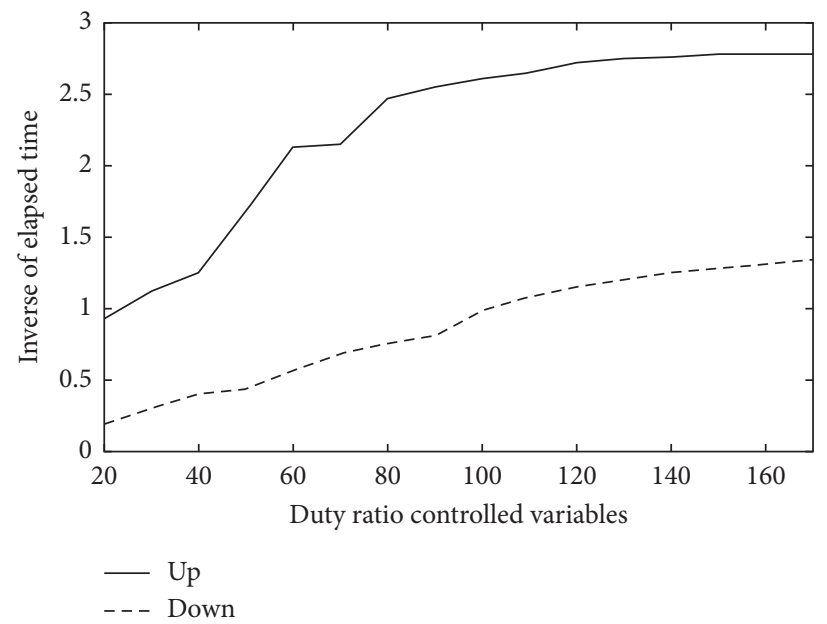

FIgURE 3: Relationship between duty-ratio-controlled variables and PAM contraction speed.

The load is gradually increased from $0 \mathrm{~kg}$ to $5 \mathrm{~kg}$ at an interval of $1 \mathrm{~kg}$. The tester is given 30 seconds of break between the two experiments to prevent muscle fatigue.
3.2. Data Processing. Data acquisition is performed throughout holding up the forearm, recording the signals derived from interception, processing, and analysis of 
experimental findings, in order to characterize the upper limb muscles' static contraction features during keeping still.

The signals may contain a lot of noise and large voltage deviation, which greatly influences real data, so it is unnecessary to filter EMG signals prior to analysis. The frequency distribution of EMGs is $20-500 \mathrm{~Hz}$, the frequency of main signals is $50-500 \mathrm{~Hz}$, and signal interception is realized by a band-pass filter with a frequency of $50-500 \mathrm{~Hz}$.

More research is needed to extract muscle contraction information after pretreatment of original EMGs. There are three methods for solving this problem: envelope, moving average, and moving RMS.

In envelope, first, full-wave rectification of the signal is performed, and then, a low band filter of two-order $2 \mathrm{~Hz}$ is applied, so that the local EMGs amplitude of the vibration can be briefly estimated.

With moving average, a period of time is selected that reads out the sum of signals after rectification $(200 \mathrm{~ms}$ is taken as research object), and then, the average is found. This can often effectively reflect the amplitude of EMGs. Generally, the longer the time chosen, the more accurate amplitude can be obtained. However, if the time is too long, it will affect real-time control, and thus, $200 \mathrm{~ms}$ is preferred.

The difference between moving RMS and moving average is in the calculation of the square root of the average.

3.3. Analysis of Results. The method of averaging the values of various quantities for 7 seconds is taken as the overall evaluation of feature variables, and the effects of load on the exoskeleton are investigated.

There are three methods of dissecting EMGs, as shown in Figures 4-7, and their results are approximately the same. The figures demonstrate how the characteristics obtained from biceps EMGs grow in an unlinear trend as more load is applied, which is closely corresponds to the EMGs and muscle force in human physiology. According to the characteristics obtained from triceps EMGs, the load comes to near-linear in the triceps. The difference between the biceps and the triceps is that the contraction length of the latter is much less than that of the former. The contraction length is very small, and the nonlinear EMG characteristics are close to linear.

\section{Dynamic Contraction of Upper Limb Muscles}

4.1. AR Model. Let $s(n)$ be a zero mean steady random signal, from which we can derive the source of white noise with known first-order and second-order statistics, where a linear system $h(k)$ is impelled by $w(n)$. The parameter $w(n)$ is zero mean white noise sequence, and its variance is $\sigma_{w}^{2}$; $h(k)$ or its transfer function $H(z)$ all comprise a linear system. If the power and system parameters of white noise excitation are known, the stochastic signal can become a model parameter, and its properties can be studied as shown in Figure 8 [16].

If $s(n)$ is only influenced by its previous value and present stimulus signals, we can obtain

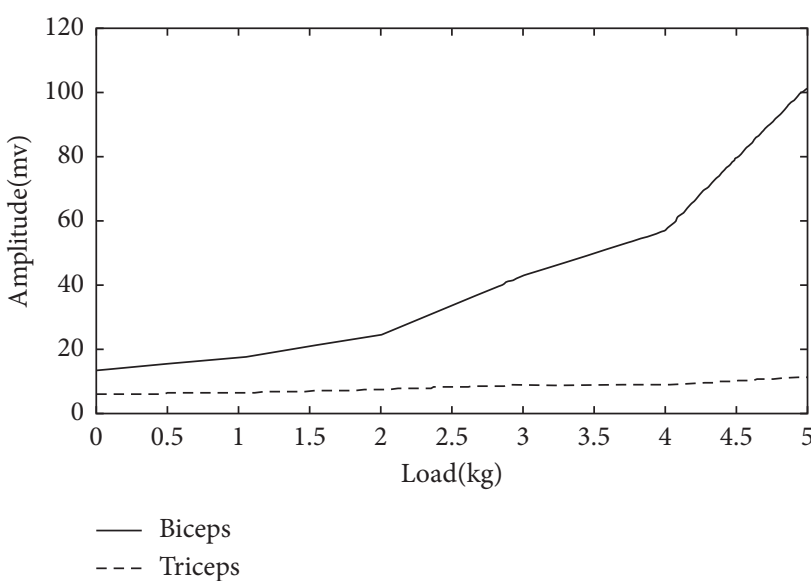

Figure 4: Biceps envelope.

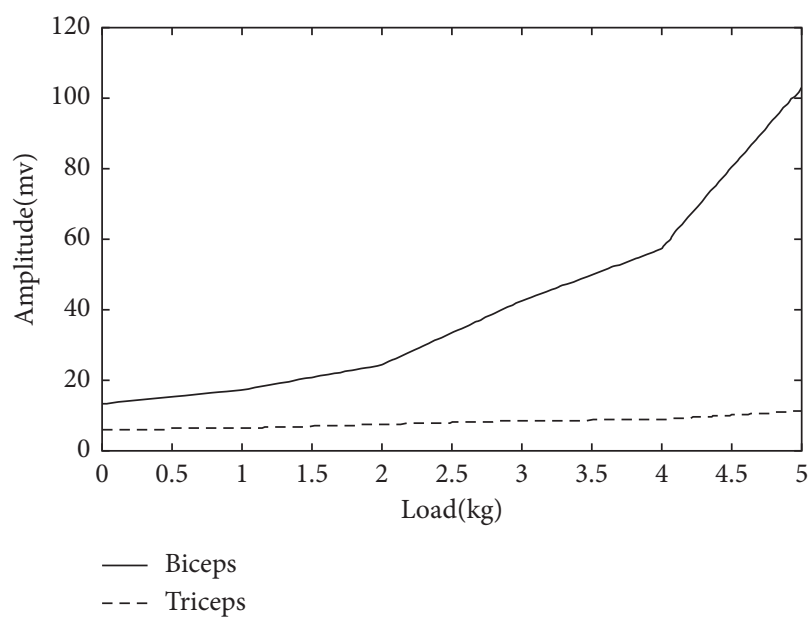

Figure 5: Biceps moving average.

$$
s(n)=-\sum_{k=1}^{p} a_{k} s(n-k)+w(n)
$$

where $a_{k}$ represents a model parameter, $p$ represents order, $w(n)$ represents zero-mean-value stationary white noise, and its variance is $\sigma^{2}$.

The model transfer function $H(z)$ can be written as

$$
\begin{aligned}
H(z) & =\frac{1}{1+\sum_{k=1}^{p} a_{k} z^{-k}} \\
& =\frac{1}{A(z)},
\end{aligned}
$$

where $A(z)=1+\sum_{k=1}^{p} a_{k} z^{-k}$.

This is a p-order all-pole AR model, in which the randomness of the stochastic signal can be combined with some predictability by model parameters. Stimulus white noise reflects the randomness of signals, while the deterministic linear model reflects signal predictability.

4.2. Module Calculation. There are many methods to estimate AR module parameters, such as the correlation method 


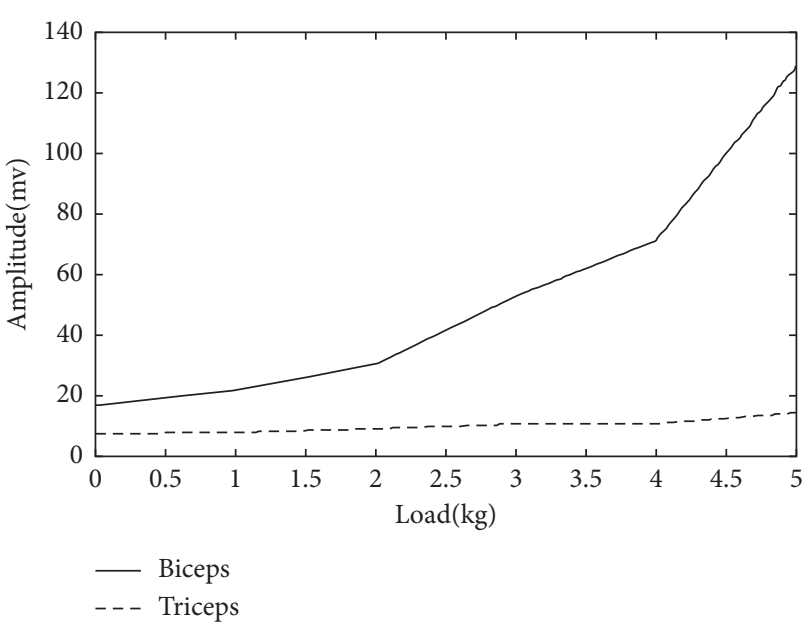

FIgURE 6: Biceps moving RMS.

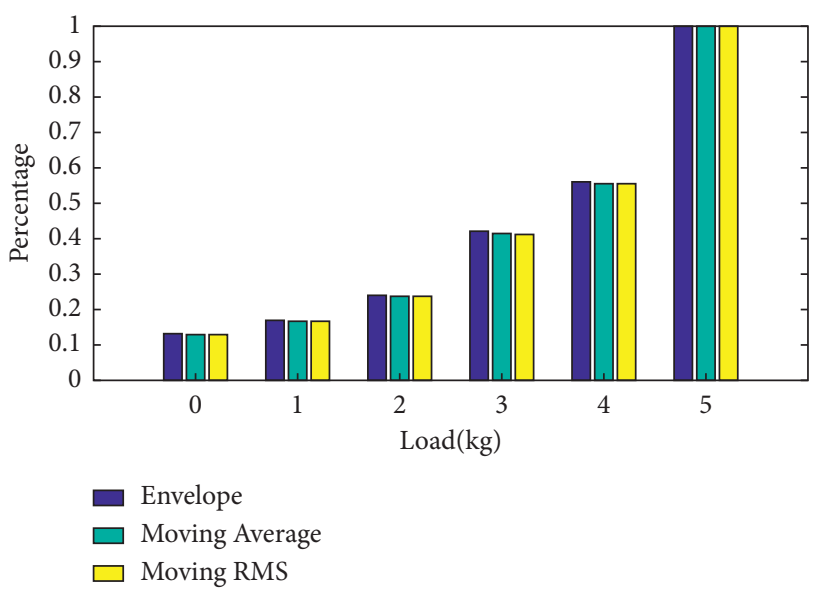

FIGURE 7: Comparison of biceps various treatment methods in the time domain.

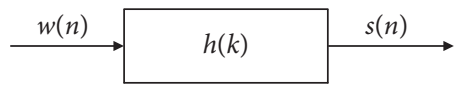

Figure 8: AR model.

and the Bury method. The relationship between AR module parameters and autocorrelation function is used in the correlation method.

For the p-order AR model, $s(n)=-\sum_{k=1}^{p} a_{k} s(n-$ $k)+w(n)$; we multiply $s(n-i)(i>0)$ on both sides of the preceding formula at the same time and then calculate the average, which is performed because $E[w(n) s(n-i)]=0$.

The Yule-Walker equation can also be deduced as

$$
R_{s s}(i)=-\sum_{k=1}^{p} a_{k} R_{s s}(i-k) i>0
$$

As can be seen from (3), for signals as described by the p-order AR model, only the $p$ autocorrelation function is independent, and the solution to the rest can be the recurrence formulas.

$$
\begin{aligned}
& \left(\begin{array}{cccc}
R_{s s}(0) & R_{s s}(-1) & \ldots & R_{s s}(1-p) \\
R_{s s}(1) & R_{s s}(0) & \ldots & R_{s s}(2-p) \\
\vdots & \vdots & \vdots & \vdots \\
R_{s s}(p-1) & R_{s s}(p-2) & \ldots & R_{s s}(0)
\end{array}\right)\left(\begin{array}{c}
a_{1} \\
a_{2} \\
\vdots \\
a_{p}
\end{array}\right) \\
& =-\left(\begin{array}{c}
R_{s s}(1) \\
R_{s s}(2) \\
\vdots \\
R_{s s}(p)
\end{array}\right) .
\end{aligned}
$$

The autocorrelation function is an even function, and the matrix is symmetric, where all diagonal elements are equal to $R_{s s}(0)$. If any elements clinodiagonal or parallel to the principal diagonal are the same, it is a Toeplitz matrix. The autocorrelation function is known, and the solution of the AR model-related coefficient is through (4).

The Burg method is the most commonly used approach for AR module parameters. It presents a method of forwarding prediction error and backward prediction error based on the least mean-square error rule, to obtain reflection coefficient $\mathrm{Km}$, which is unexpectedly left outside of known data. The Burg method demonstrates better resolution than the correlation method on this account and is the proper solution for analyzing EMGs.

4.3. Feature Extraction. Using the copy of data analyzed in AR module parameters, we carry out the division to EMGs after filtering. The longer the segment of data, the greater meaning it contains, and the higher stability and accuracy of the AR coefficient. However, if the segment of data is too long, it is not conducive to real-time information control. To guarantee real-time control, and to avoid causing a discernible delay, the total time for data storage and data processing cannot be more than $300 \mathrm{~ms}$.

There are two alternative segmentation methods, namely, overlapping window and alternating windows.

In the overlapping window method, operations are constantly performed on data, the same block of data is executed for a specified number of times, the more running code is operated, and it will be more complicated for the control system. If accelerated by computing, the data processing time can be reduced to $1 \mathrm{~ms}$, and a good result for the data segment of $200 \mathrm{~ms}$ can be obtained. Taking all factors into consideration, alternating windows are chosen for segmentation.

As can be derived from Figures 9-11, with the increase of amplitude, the AR module coefficient enhances, and the order coefficient does not change much. If the sum of the absolute value of the AR module order is directly adopted, the other items will not adjust automatically, and it increases with load.

4.4. Forearm Rapid Swing and Slow Swing. With the forearm held up and the elbow joint rotated, the EMGs of biceps and triceps change are explored under different loads. 

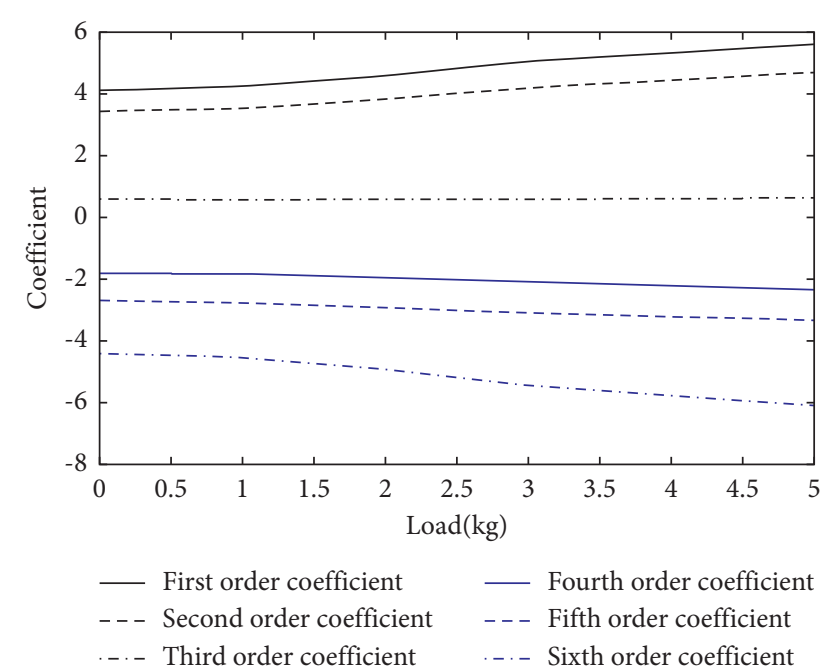

FIgURE 9: Change of biceps AR module coefficient.

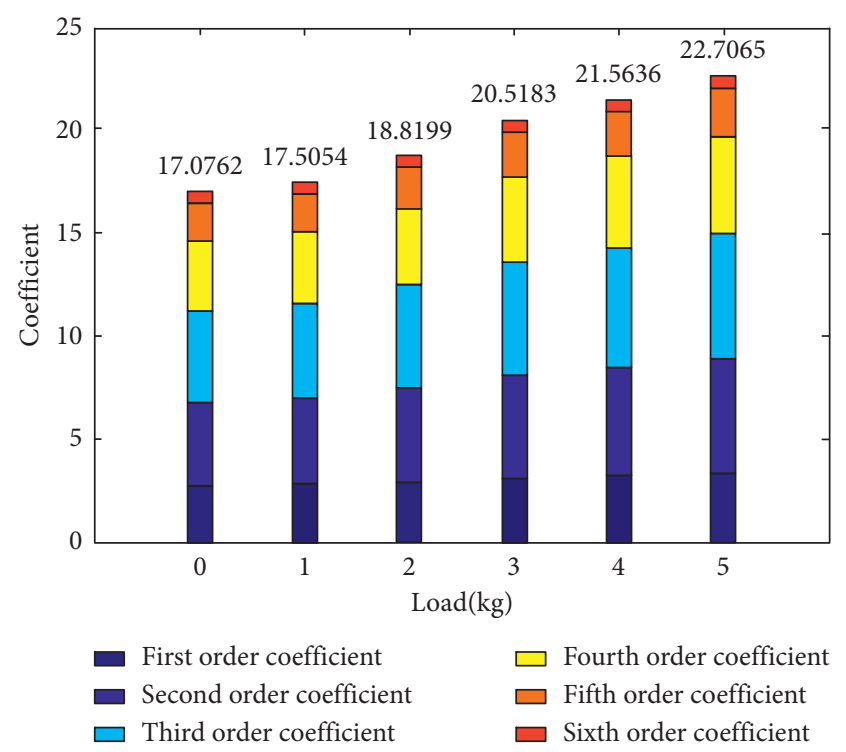

FIgURE 10: Absolute value sum of biceps AR module coefficient.

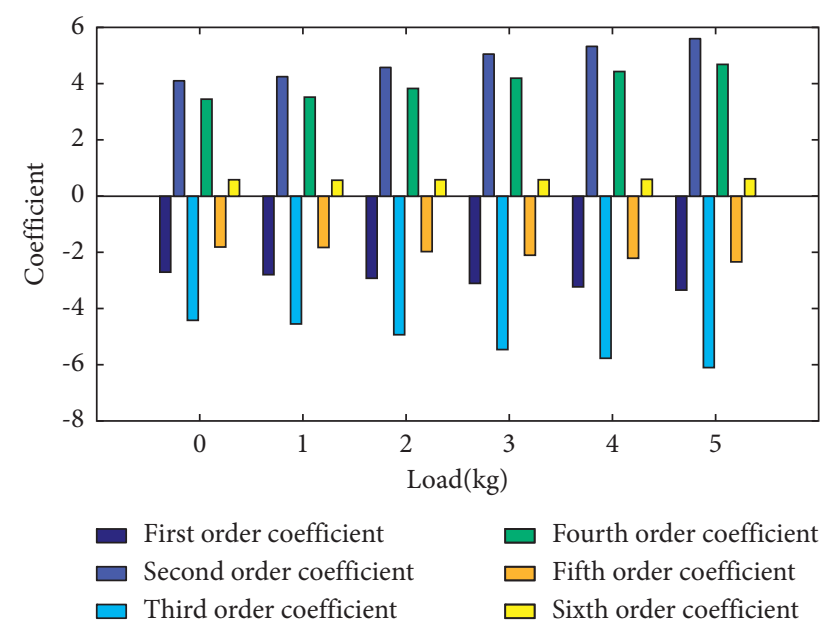

FIgURE 11: Biceps AR module coefficient.
The EMGs amplitude can be estimated by the methods of envelope, moving average, and moving RMS with the static contraction of upper limb muscles. However, the method of envelope provides much higher real-time amplitudes, which is of great benefit to the control system design, and is selected to obtain the upper limb muscle dynamic contraction feature variables.

The tester lifts their forearm as briskly as they can with the loaded exoskeleton in rapid swing, while it is lifted slowly in slow swing.

As shown in Figure 12, biceps EMGs are the most obvious, and the amplitude of triceps EMGs rises and falls, but at a modest rate when the forearm is held up. Biceps EMGs rapidly increase with the elbow joint angle, decay quickly as the elbow joint angle remains unchanged, and continue to fall if the angle decreases. This suggests that rapid swing occurs at the moment when the forearm outputs force when biceps EMGs gain more intensity and quickly fall back. The intensity of biceps EMGs increases slowly, and it fluctuates during the forearm slow swing. The signal intensity during rapid swing is much higher than that during slow swing.

4.5. Intermittent Forearm Swing. The movement of the upper limb is usually irregular, not always showing reciprocating action and featuring spasmodic stops in usual daily life. Therefore, the value changes of EMGs in such cases will have to be studied. Here, the exoskeleton is loaded with $3 \mathrm{~kg}$ weight, and the tester lifts-up their forearm freely.

As shown in Figure 13, the relationship between EMGs intensity and joint angle is nonlinear. The amplitude of EMGs does not increase when the angle is $0-50^{\circ}$. However, it obviously increases when the angle is $60-85^{\circ}$. In addition, when the angle changes abruptly, the amplitude also changes suddenly.

4.6. Comparison of Results. The large difference between dynamic contraction and static contraction is that EMGs reach the peak at one moment, and the highest value has nothing to do with joint velocity change. The change in joint velocity is fast, the muscular instantaneous contraction is violent, and EMGs are intense. The analysis of maximum EMGs can reflect the dynamic contraction of muscles.

Table 1 and Figure 14 show that there is a distinctive difference between rapid swing and slow swing in rise time and fall time, which further effectively proves that movement modes can be classified into rapid and slow swing in dynamic contraction. The amplitude of maximum EMGs increases with the load, whether rapid swing or slow swing, which is similar to static contraction. Meanwhile, it is obvious that the relationship between maximum EMGs amplitude and load is nonlinear; the growth rate is comparatively larger at the beginning and then slowly descends. Moreover, by comparing the two different swing modes, we can find that the capacity of separating the maximum EMGs is weak when the load is small, and EMGs' magnitude for fast swing is much larger than that for slow swing when the load is large. 

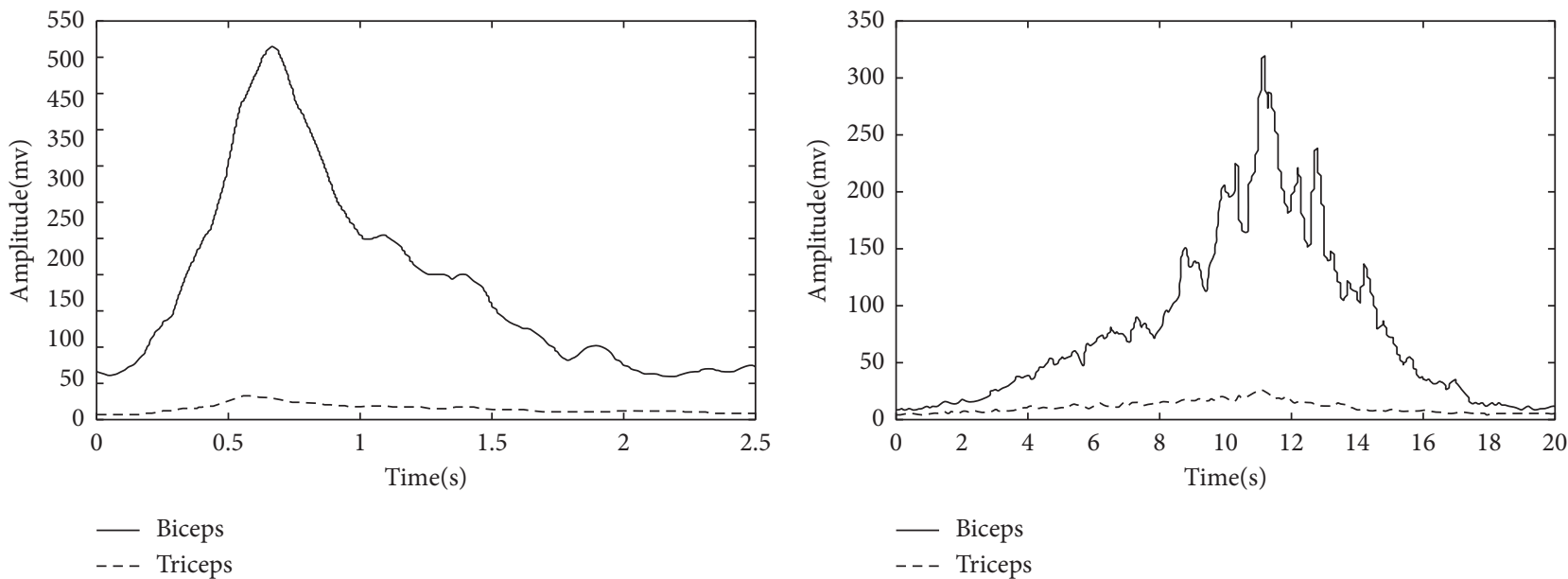

(a)

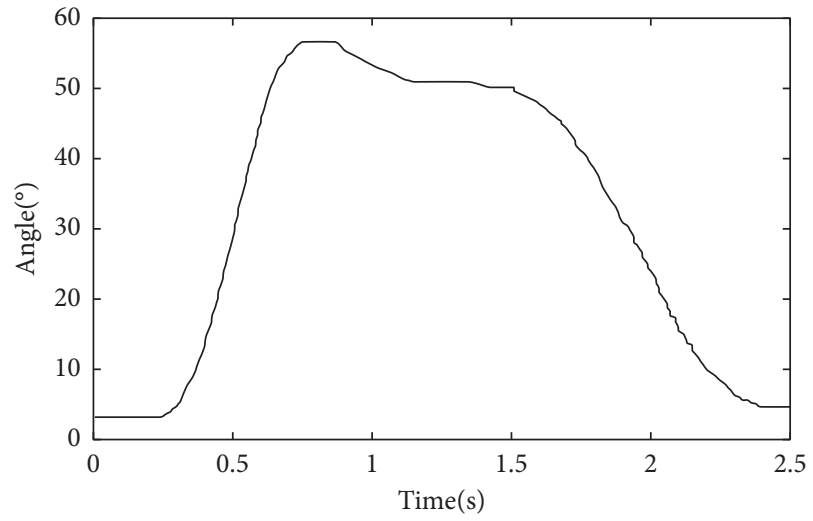

(b)

(c)

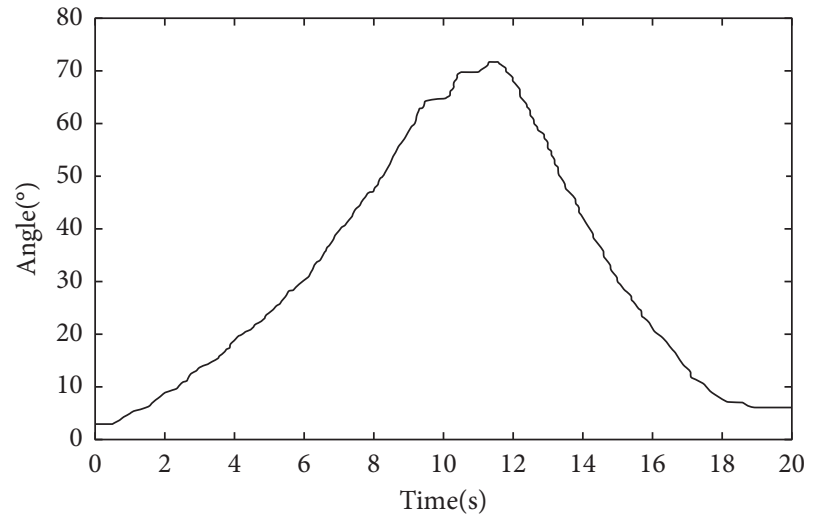

(d)

FIGURE 12: Dynamic contraction of (a) EMGs featuring value change in rapid swing, (b) EMGs featuring value change in slow swing, (c) elbow joint change in rapid swing, (d) elbow joint change in slow swing, with $5 \mathrm{~kg}$ load.

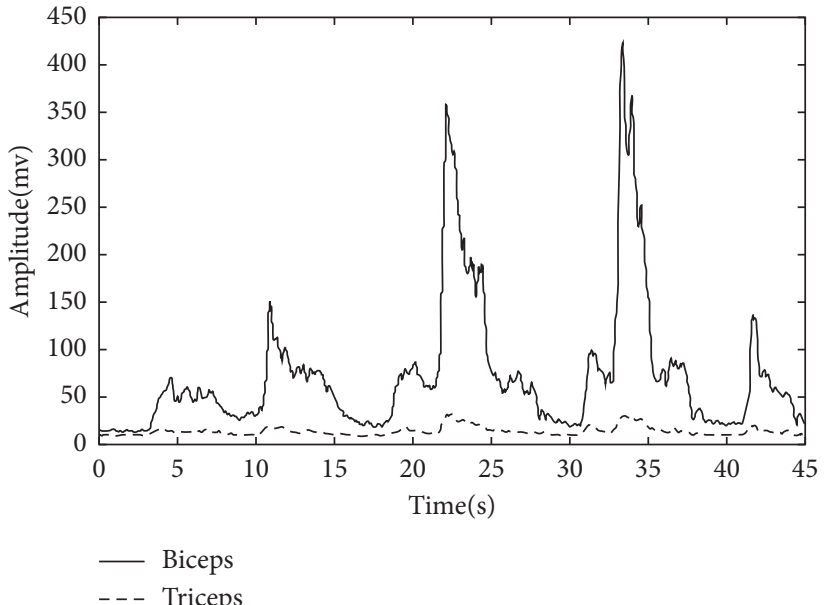

(a)

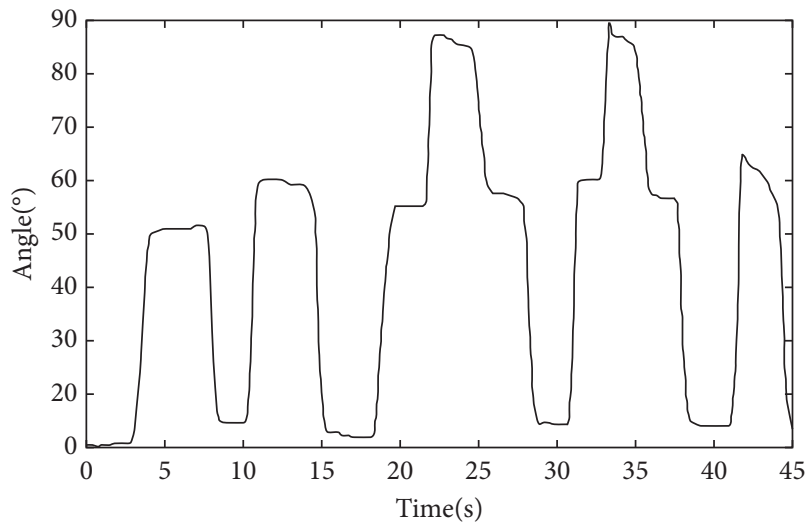

(b)

FIGURE 13: Discontinuous swing; (a) EMGs feature value change in discontinuous swing and (b) elbow joint change in discontinuous swing, with $3 \mathrm{~kg}$ load applied. 
TABLE 1: Comparison of dynamic contraction under various loads.

\begin{tabular}{|c|c|c|c|c|c|c|}
\hline \multirow[t]{2}{*}{ Load (kg) } & \multicolumn{2}{|c|}{ Rise time (s) } & \multicolumn{2}{|c|}{ Fall time (s) } & \multicolumn{2}{|c|}{$\begin{array}{l}\text { Maximum } \\
\text { amplitude } \\
(\mathrm{mV})\end{array}$} \\
\hline & Rapid & Slow & Rapid & Slow & Rapid & Slow \\
\hline 0 & 0.5 & 10 & 0.5 & 12 & 130 & 80 \\
\hline 1 & 0.5 & 10 & 0.6 & 18 & 180 & 140 \\
\hline 2 & 0.5 & 15 & 0.8 & 10 & 300 & 250 \\
\hline 3 & 0.5 & 10 & 0.8 & 8 & 480 & 270 \\
\hline 4 & 0.5 & 10 & 1 & 8 & 485 & 310 \\
\hline 5 & 0.5 & 9 & 1 & 6 & 514 & 325 \\
\hline
\end{tabular}

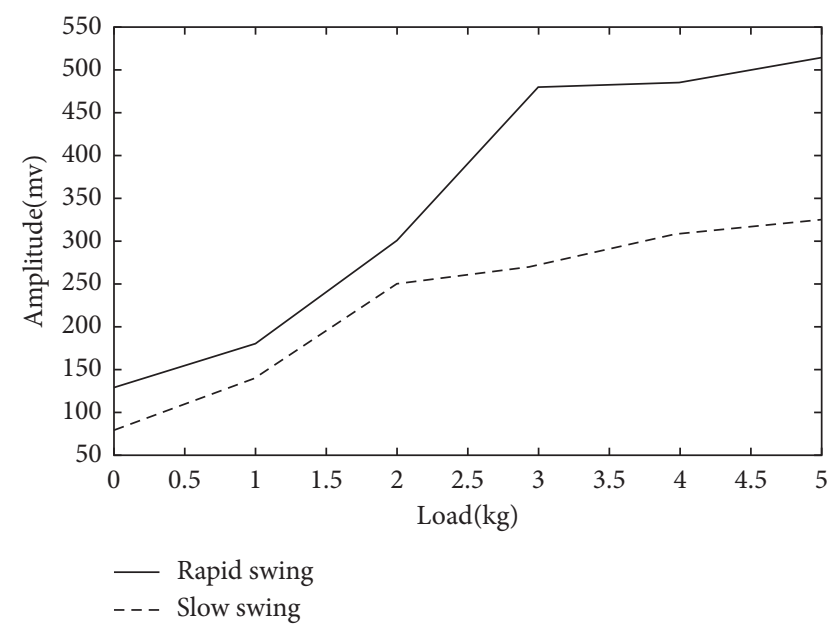

FIgURe 14: Maximum EMG under various loads.

\section{Man-Machine Synergy Control}

5.1. EMGs Mapping Relationships. When the tester is in the exoskeleton, muscle force can be subjectively conceived into five levels: powerless, weak, moderate, strong, and very strong, and its corresponding duty-ratio-controlled variables are 20, 40,60,80, and 100. The biceps (pulling up) and triceps (pulling down) EMGs strength can be estimated in such a case. The method for EMGs strength calculation is to average the EMGs characters of muscle force within 0.5 seconds.

$$
\overline{\mathrm{EMG}}=\frac{\sum_{i=0}^{1000} \mathrm{EMG}(i)}{1000},
$$

where $\mathrm{EMG}(i)$ represents sampled data, and $\overline{\mathrm{EMG}}$ is the average of EMGs.

The sampled data are shown in Table 2.

From Figure 15, a nonlinear relationship between dutyratio-controlled variables and average EMG characters can be derived. This suggests that EMGs generated by human perception of muscle force are nonlinear. If the exoskeleton is in strict accordance with linear control, we will quickly run into rotational speed increases with the elbow joint. Thus, it is easily operated, and the operation is linear control in human perception, and nonlinear EMGs are mapped to duty-ratio-controlled variables.
There are only 5 separated points in Figure 15 . The average EMG characters can be contrasted with the dutyratio-controlled variables, and the two appear in a continuum for continuous control; hence, interpolation is needed for the 5 separated points.

5.2. Polynomial Interpolation. A series of points also looks as an approximate expression when using interpolation. The choices of functional form can be various, such as trigonometric function and rational function. Algebraic polynomial interpolation is applied, as it is easily performed by the function of $f(x)=a_{0}+a_{1} x+a_{2} x^{2}+\ldots+a_{n} x^{n}$.

As the function has 5 points, according to the polynomial interpolation uniqueness condition, the fourth-order polynomial is satisfactory. Its key is to define the related coefficient of the polynomial function. There are many methods to solve the problem, such as multivariable polynomial system or Lagrangian method. The order of interpolation is small, and it is easy to find a solution for equations. We can obtain the polynomial coefficient by developing programs directly for equations in software, and the procedure is presented below.

There are 5 points, namely, $\left(x_{0}, y_{0}\right),\left(x_{1}, y_{1}\right),\left(x_{2}, y_{2}\right)$, $\left(x_{3}, y_{3}\right)$, and $\left(x_{4}, y_{4}\right)$, which satisfy the function of $f(x)=a_{0}+a_{1} x+a_{2} x^{2}+a_{3} x^{3}+a_{4} x^{4}$, giving the following equations [17]:

$$
\left\{\begin{array}{l}
a_{0}+a_{1} x_{0}+a_{2} x_{0}^{2}+a_{3} x_{0}^{3}+a_{4} x_{0}^{4}=y_{0}, \\
a_{0}+a_{1} x_{1}+a_{2} x_{1}^{2}+a_{3} x_{1}^{3}+a_{4} x_{1}^{4}=y_{1}, \\
a_{0}+a_{1} x_{2}+a_{2} x_{2}^{2}+a_{3} x_{2}^{3}+a_{4} x_{2}^{4}=y_{2}, \\
a_{0}+a_{1} x_{3}+a_{2} x_{3}^{2}+a_{3} x_{3}^{3}+a_{4} x_{3}^{4}=y_{3}, \\
a_{0}+a_{1} x_{4}+a_{2} x_{4}^{2}+a_{3} x_{4}^{3}+a_{4} x_{4}^{4}=y_{4}
\end{array}\right.
$$

Using the Gaussian elimination method of linear algebra, the combination of elimination method and recursive method as a solution to the above equations can yield the values of 5 parameters.

Given the parameters in Table 3 above as a reference, the mapping relation function image is illustrated in Figure 16.

As can be seen in Figure 16, the interpolation function goes through points that are detected using the interpolation method, and ideal nonlinear mapping can be realized around several measured points. These points easily fluctuate around the measured points. According to the triceps mapping relation in Figure 16, the average EMGs character is more than 50, and the corresponding duty-ratio-controlled variables exhibit a sharp drop, which is even negative.

This explains the problem of man-machine synergy control. The operator's upper limb swings up, stops in midstream, and then swings up again, which will lead to the upper limb's shaking. This is mainly because of a sudden change of the moving direction of human muscle dynamic contraction, causing a sharp maximum in EMGs. The dutyratio-controlled variables of the triceps will offset those of the biceps first, and then, triceps EMGs are in the process of 50-80, falling off sharply. Subsequently, the net duty-ratiocontrolled variables increase again, which causes vibration. 
TABLE 2: Exertion rating and average EMGs characters.

\begin{tabular}{lccc}
\hline Exertion rating & Duty-ratio-controlled variables & Biceps EMG ratio & Triceps EMG ratio \\
\hline Powerless & 20 & 11.9 & 4.8 \\
Weak & 40 & 21.3 & 9.7 \\
Moderate & 60 & 33.0 & 18.3 \\
Strong & 80 & 44.1 & 29.4 \\
Very strong & 100 & 79.1 & 49.4 \\
\hline
\end{tabular}

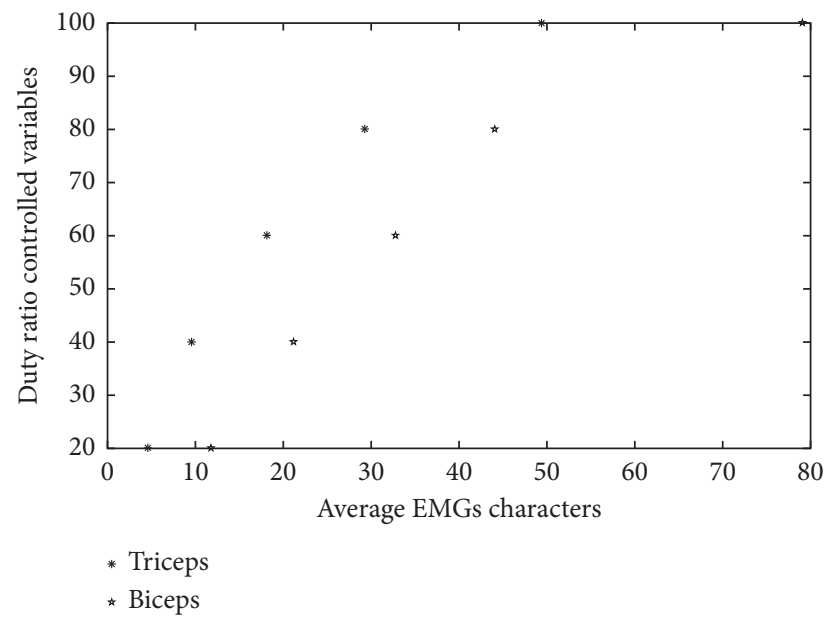

FIGURE 15: Scatter diagram of duty-ratio-controlled variables and average EMG characters.

TABLE 3: Mapping relation parameters.

\begin{tabular}{|c|c|c|c|c|c|}
\hline Signal source & $a_{0}$ & $a_{1}$ & $a_{2}$ & $a_{3}$ & $a_{4}$ \\
\hline Biceps & -21.810601 & 4.827553 & -0.138556 & 0.002629 & -0.000018 \\
\hline Triceps & -11.075030 & 8.138877 & -0.382913 & 0.009916 & -0.000093 \\
\hline
\end{tabular}

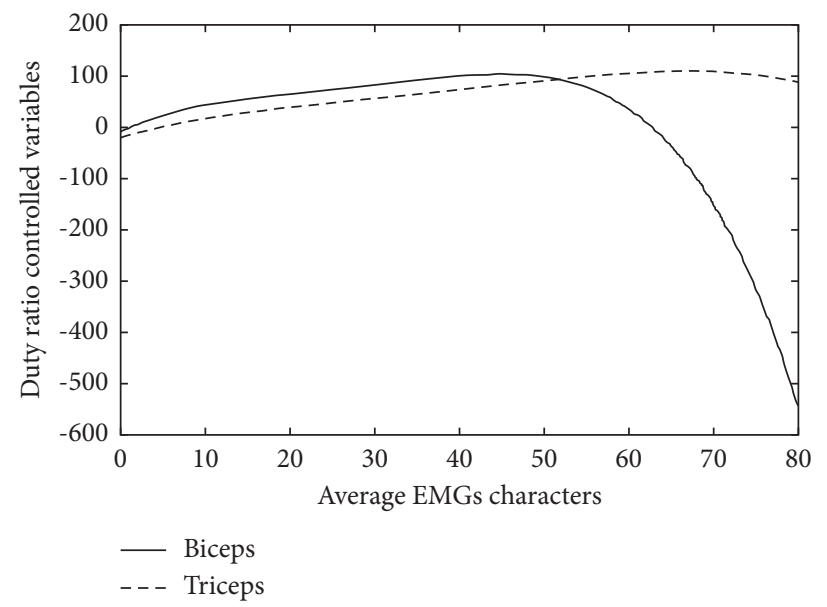

Figure 16: Duty-ratio-controlled variables and average EMGs characters.

To solve the problem of EMGs mapping uncertainty outside the range of interpolation, the answer is to cut out the EMGs' feature value. The oversized EMGs feature value matches the maximal duty-ratio-controlled variables directly. When EMGs are very small, the duty-ratio-controlled variables are set to the high-speed on-off valve dead zone critical point, which is 15 .
5.3. Determination of Synergic Control Method. The synergic control method falls into three steps: feature extraction, nonlinear mapping, and comparison judgment.

Feature extraction only consists of the high-pass filter, full-wave rectifier, and low-pass filter, originally acquiring the EMGs data of two motions, the envelope curve of biceps and triceps EMGs, which are $\mathrm{EMG}_{1}$ and $\mathrm{EMG}_{2}$, respectively. 
According to the method claimed above, the biceps and triceps ratings of perceived exertion average EMGs characters are $\mathrm{EMG}_{10} \ldots \mathrm{EMG}_{14}$ and $\mathrm{EMG}_{20} \ldots \mathrm{EMG}_{24}$, respectively, and then, in polynomial interpolation, the interpolation function coefficients are $a_{10} \ldots a_{14}$ and $a_{20} \ldots a_{24}$, and the mapping function is as follows:

$$
\begin{aligned}
& \text { Duty }_{\mathrm{emg} 1}(x)= \begin{cases}15, & x \leq \mathrm{EMG}_{10}, \\
a_{10}+a_{11} x+a_{12} x^{2}+a_{13} x^{3}+a_{14} x^{4}, & \mathrm{EMG}_{10}<x<\mathrm{EMG}_{14}, \\
100, & x \geq \mathrm{EMG}_{14},\end{cases} \\
& \text { Duty }_{\mathrm{emg} 2}(x)= \begin{cases}15, & x \leq \mathrm{EMG}_{20}, \\
a_{20}+a_{21} x+a_{22} x^{2}+a_{23} x^{3}+a_{24} x^{4}, & \mathrm{EMG}_{20}<x<\mathrm{EMG}_{24}, \\
100, & x \geq \mathrm{EMG}_{24} .\end{cases}
\end{aligned}
$$

After the nonlinear mapping of EMGs, the net dutyratio-controlled variables Duty net can be calculated as

$$
\text { Duty }_{\text {net }}=\text { Duty }_{\mathrm{emg} 1}\left(\mathrm{EMG}_{1}\right)-\text { Duty }_{\mathrm{emg} 2}\left(\mathrm{EMG}_{2}\right) \text {, }
$$

where Duty $_{\mathrm{emg} 1}\left(\mathrm{EMG}_{1}\right)$ and Duty $\mathrm{emg}_{2}\left(\mathrm{EMG}_{2}\right)$ are the dutyratio-controlled variables of the first and second high-speed on-off valve, respectively.

\subsection{Forearm Lift-Up Motion When Unpowered with Various} Loads. The biceps and triceps EMGs are taken as control input when the tester is in the exoskeleton while studying the load controlling results and the effect of weight reduction by the exoskeleton.

The experiment includes two parts: forearm EMGs unpowered with various loads and forearm EMGs powered by the exoskeleton with various loads.

The tester stands still, the surface electrodes are pasted to a relevant location outside of the biceps and triceps, the tester outputs upward force, and the exoskeleton swings up, recording EMGs and the angle in the whole process.

It can be derived from Figure 17 and 18 that the biceps EMGs increase with elbow joint angle and triceps EMGs change little in the process.

\subsection{Forearm Lift-Up Motion When Powered with Various} Loads. The EMGs and angle joint variation curves (powered, $5 \mathrm{~kg}$ ) can be seen in Figures 19 and 20. When the operator lifts-up and puts down the forearm, muscles are activated. The movements are biceps upward and triceps downward, and the essential distinction is obvious, which is different from the powered condition. The load is not on the operator when powered, and the biceps does not need to be in tension all the time during lifting up and putting down the forearm; there is a great deal of difference in EMGs change. The wave deformation is very similar in the process of lifting up when powered, and the variance of maximum EMGs is much larger than the average EMGs.

5.6. Maximum EMGs Powered Efficiency. The average EMGs feature value describes EMGs in the whole process, and the maximum EMGs feature value describes the extent to which the muscles are activated during dynamic muscle contraction. The biceps maximum EMGs in lifting up under unpowered and powered conditions are compared to evaluate powered efficiency.

The maximum EMGs powered efficiency can be derived from Table 4. The maximum value of EMGs shows a sharp reduction between unpowered and powered condition, and the maximum EMGs powered efficiency can reach $78.66 \%$. The less the maximum EMGs, the more comfortable for the operator, and the relatively smaller transient impact to the limb.

5.7. Powered Stability Analysis. The intensity of EMGs rises with the increase of load when unpowered; however, the muscle output force is approximating similar without much fluctuation when powered. To evaluate the fluctuation of the control system relative to load change, EMGs mean variance is calculated for various loads.

Table 5 describes powered stability. The mean of biceps EMGs is 26.05 during lifting up with the load increased from $0 \mathrm{~kg}$ to $10 \mathrm{~kg}$, which is smaller than that for an unpowered condition without any load, and the standard deviation is 3.79. The mean of triceps EMGs is 19.01 during putting down, while the unpowered minimum biceps feature value is 25.3. With the increase of load, the force change is very small for the operator to control the exoskeleton, and the results for exoskeleton assistance are excellent.

5.8. Coordination Effect Analysis. In order to evaluate the advantage of the control method, man-machine synergy control is another aspect besides assistance. The aim of synergy control is that man should be synchronized with the exoskeleton, and the operator can achieve starting and stopping the control of the exoskeleton.

The discontinuous swing EMGs and angle joint variation curves are shown in Figures 21 and 22. The operator stops the exoskeleton midstream, and there is an angle overshoot, which diminishes gradually with the load increase and inertia moment. 


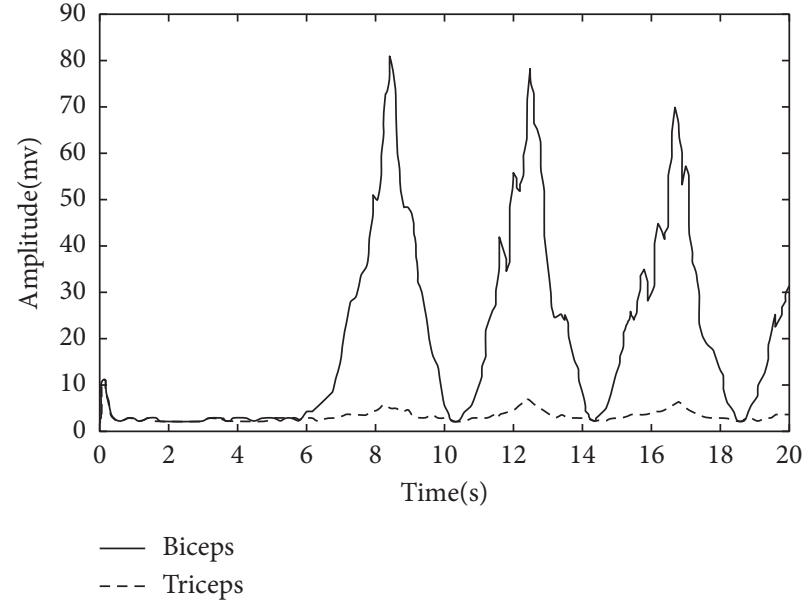

(a)

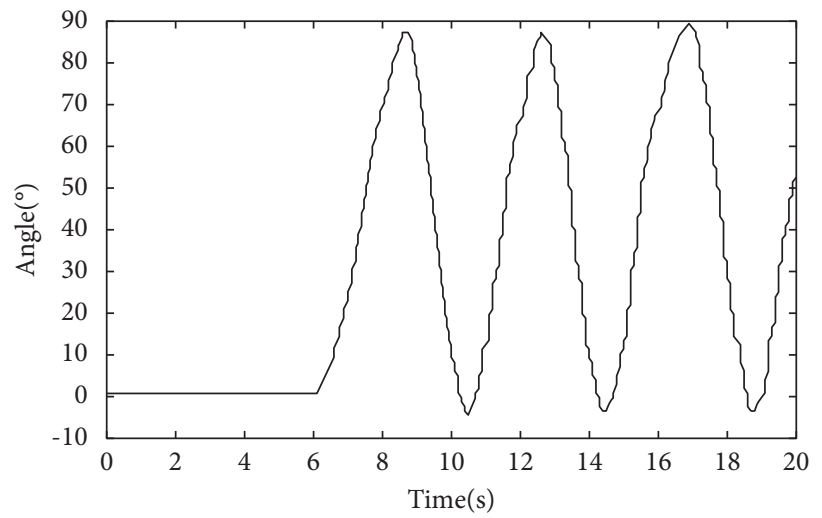

(b)

FIGURE 17: EMGs and angle joint variation curves of (a) EMGs and (b) angle joint; unpowered (3 kg).
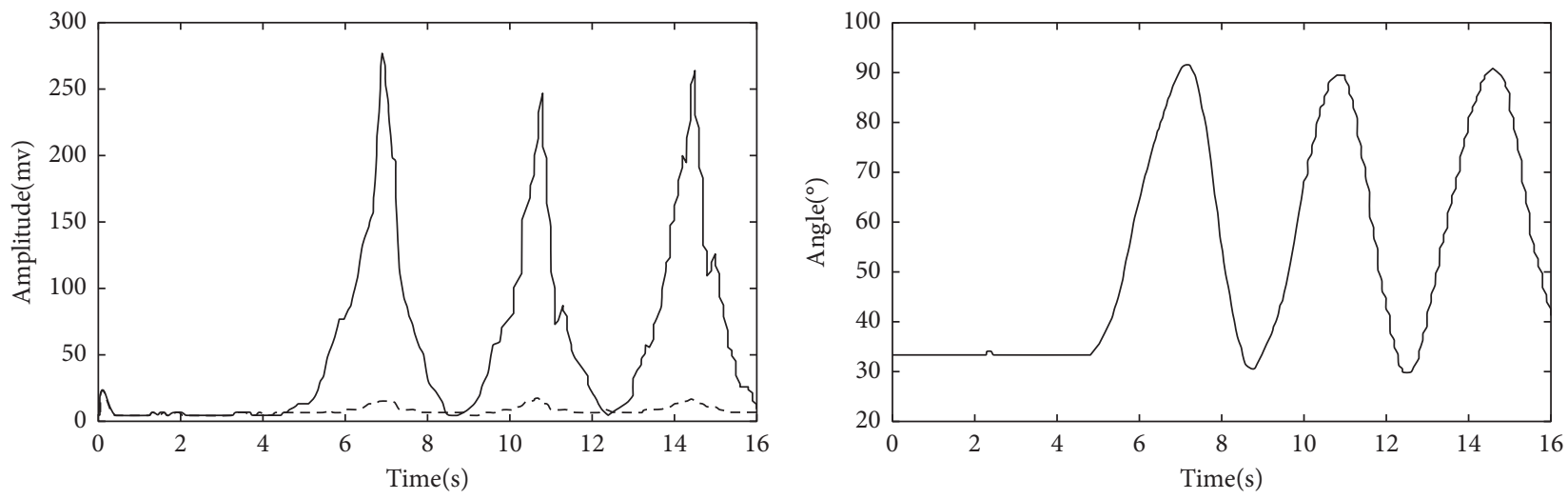

- Biceps

-._ Triceps

(a)

(b)

FIGURE 18: EMGs and angle joint variation curves of (a) EMGs and (b) angle joint; unpowered (5 kg).

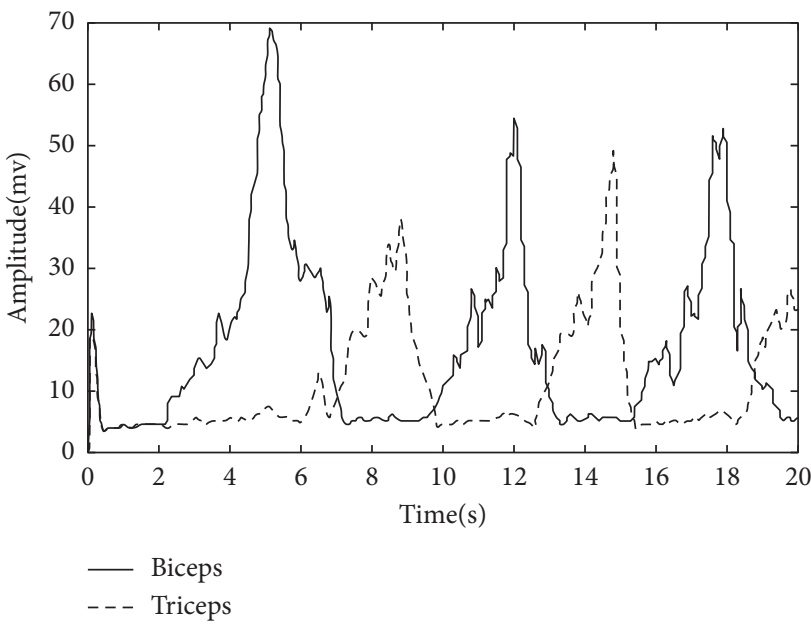

(a)

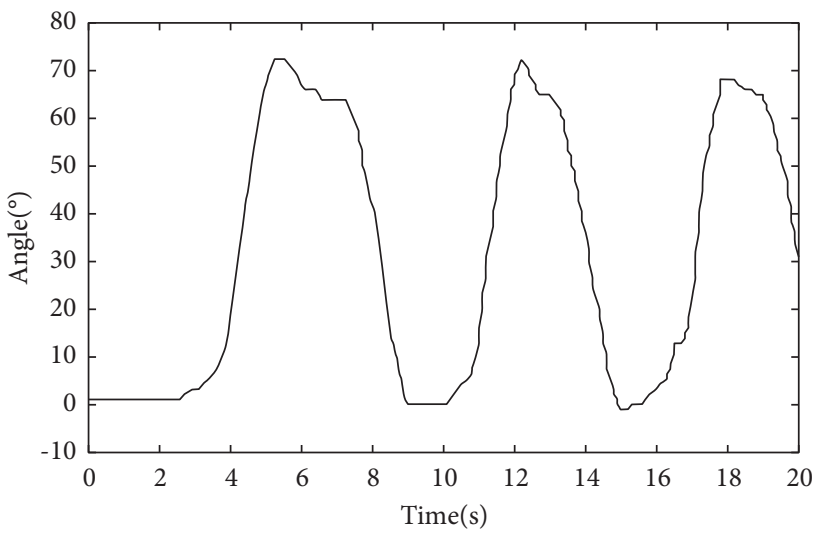

(b)

FIGURE 19: EMGs and angle joint variation curves of (a) EMGs and (b) angle joint; powered condition (3 kg). 


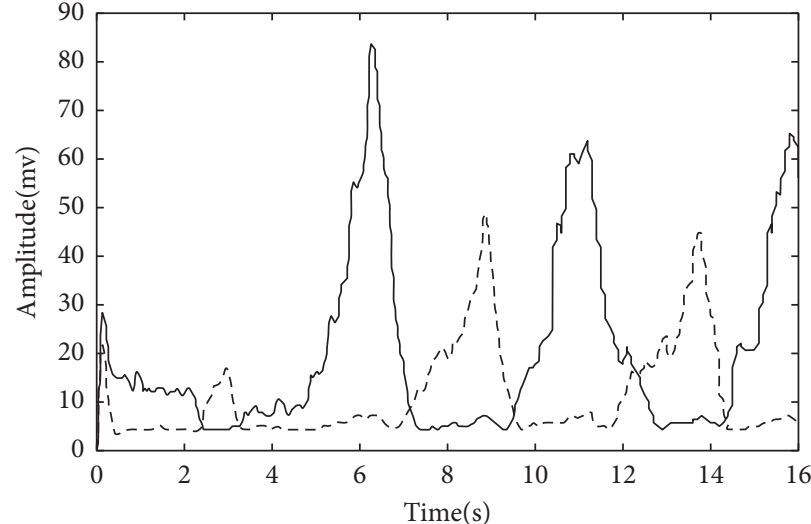

- Biceps

-._ Triceps

(a)

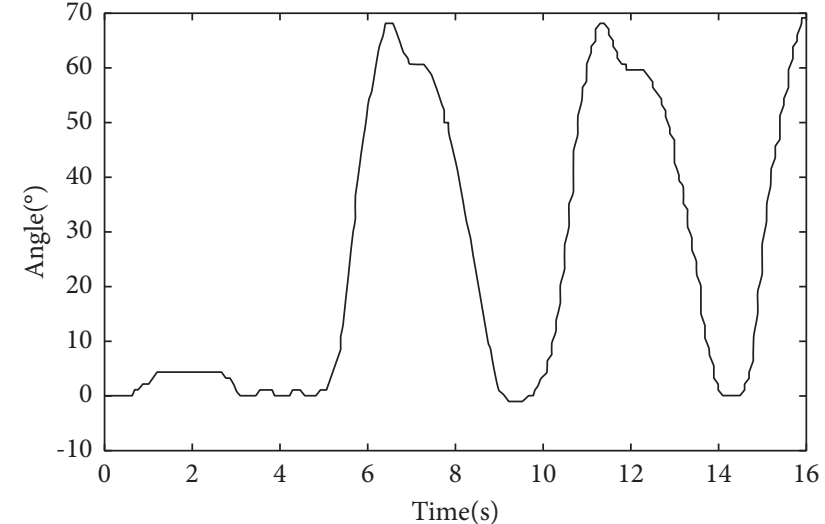

(b)

FIGURE 20: EMGs and angle joint variation curves of (a) EMGs and (b) angle joint; powered condition (5 kg).

TABle 4: Maximum EMGs powered efficiency.

\begin{tabular}{lccc}
\hline Load $(\mathrm{kg})$ & & Lifting up & \\
Powered & Powered efficiency \\
\hline 0 & Unpowered & 72.1 & 2.57 \\
1 & 74.0 & 50.9 & 48.22 \\
2 & 98.3 & 64.1 & 41.73 \\
3 & 110 & 53.8 & 65.51 \\
4 & 156 & 55.9 & 78.66 \\
5 & 262 & 63.2 & 76.93 \\
\hline
\end{tabular}

TABle 5: Powered stability.

\begin{tabular}{lcccc}
\hline Statistics & Lifting up & \multicolumn{2}{c}{ Putting down } \\
& Biceps & Triceps & Biceps & Triceps \\
\hline Average value & 26.05 & 5.39 & 9.22 & 19.01 \\
Variance & 3.79 & 0.30 & 1.48 & 3.97 \\
\hline
\end{tabular}

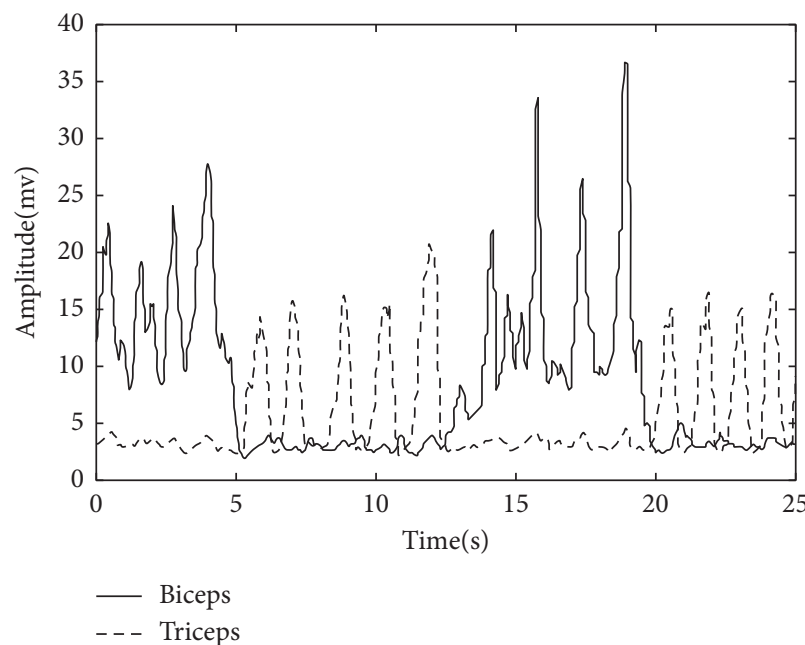

(a)

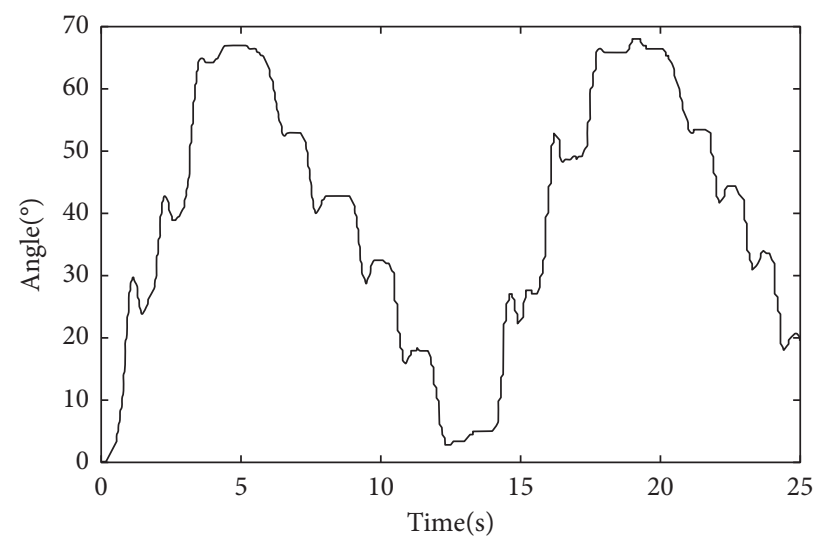

(b)

Figure 21: Discontinuous swing EMGs and angle joint variation curves of (a) EMGs, (b) angle joint under $3 \mathrm{~kg}$ load. 


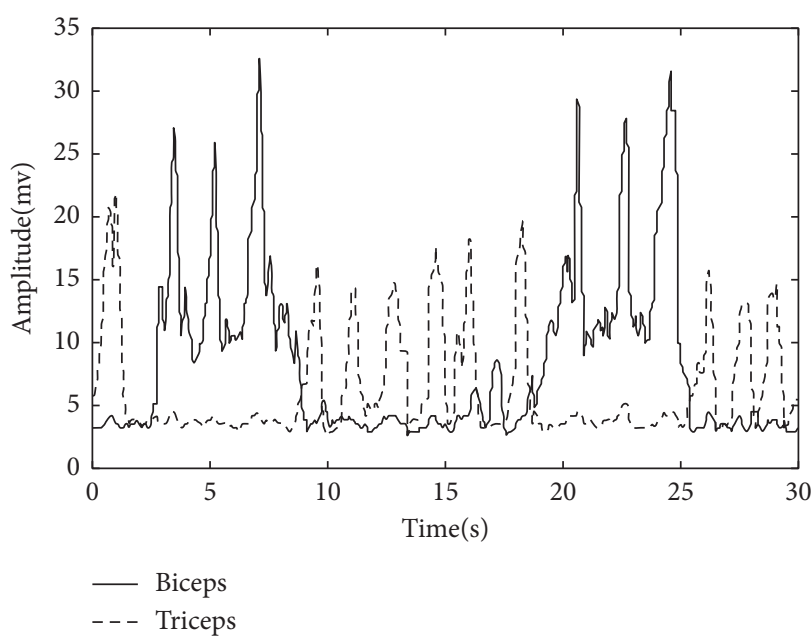

(a)

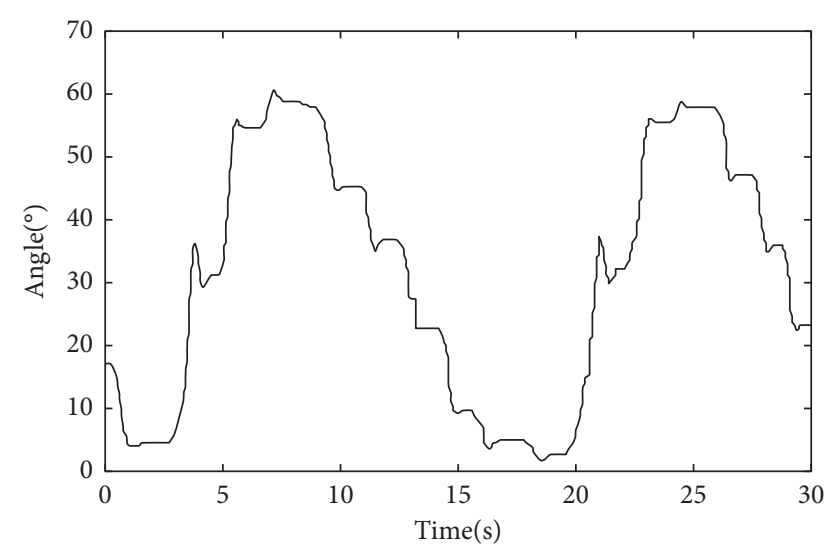

(b)

Figure 22: Discontinuous swing EMGs and angle joint variation curves of (a) EMGs, (b) angle joint under $5 \mathrm{~kg}$ load.

\section{Conclusions}

This study analyzed the amplitude and frequency characteristics of EMGs and proposed a suitable signal processing scheme. The acquisition instrument was selected, and the EMGs acquisition system was established. The biceps and triceps EMGs static contraction and dynamic contraction, and rotational speeds were analyzed under various loads ( $3 \mathrm{~kg}$ and $5 \mathrm{~kg}$, respectively). The duty-ratiocontrolled variables could be divided into five levels corresponding to the exertion rating from powerless to very strong in two EMGs characters, which can be reflected in the scatter diagram of duty-ratio-controlled variables and average EMGs characters. The net dutyratio-controlled variables were adopted to control the onoff state and PWM duty ratio of the high-speed on-off valve. The maximum EMGs powered efficiency, powered stability analysis, and coordination effect all indicate that the pneumatic-powered exoskeleton is of good assistance, and synergic control also works perfectly in forearm liftup movement powered under various loads, whether the elbow is in continuous swing or not.

We may extend the proposed methods to uncertain probabilistic linguistic environments in our future studies $[18,19]$, including decision-making with probabilistic hesitant fuzzy information based on multiplicative consistency, instead of the man-machine synergic control in a specific environment. We may also extend the proposed methods to probabilistic linguistic uncertain environments in our future studies, such as score function based on concentration degree for probabilistic linguistic term sets, evaluating the Internet of Things platforms using integrated probabilistic linguistic multicriteria decisionmaking method [20-23]. We can also introduce a nonlinear control algorithm in the controller design, such as fuzzy control, neural network control [24], and observerbased fuzzy adaptive control [25].

\section{Data Availability}

All data, models, or code generated or used to support the findings of this study are available from the corresponding author upon request.

\section{Conflicts of Interest}

The author(s) declare no potential conflicts of interest with respect to research, authorship, and/or publication of this article.

\section{Acknowledgments}

This project was supported by the Zhejiang Province Welfare Technology Applied Research Project (project no. GLF 19E050003), College Students' Science and Technology Innovation and XinMiao Project of Zhejiang Province (project no. 2021R417019), and Zhejiang Province Welfare Technology Applied Research Project (project no. GLF 20E090006).

\section{References}

[1] R. F. Antonio, L. P. Joan, and L. P. Josep, "Systematic review on wearable lower-limb exoskeletons for gait training in neuromuscular impairments," Journal of NeuroEngineering and Rehabilitation, vol. 18, p. 22, 2021.

[2] J. Narayan, B. Kalita, and S. K. Dwivedy, "Development of robot-based upper limb devices for rehabilitation purposes: a systematic review," Augmented Human Research, vol. 6, no. 1, p. 4, 2021.

[3] M. Dong, Y. Zhou, J. Li et al., "State of the art in parallel ankle rehabilitation robot: a systematic review," Journal of NeuroEngineering and Rehabilitation, vol. 18, no. 1, p. 52, 2021.

[4] N. P. Fromme, M. Camenzind, R. Riener, and R. M. Rossi, "Need for mechanically and ergonomically enhanced tremorsuppression orthoses for the upper limb: a systematic review," 
Journal of NeuroEngineering and Rehabilitation, vol. 16, no. 1, p. 93, 2019.

[5] C. Lambelet, D. Temiraliuly, M. Siegenthaler et al., "Characterization and wearability evaluation of a fully portable wrist exoskeleton for unsupervised training after stroke," Journal of NeuroEngineering and Rehabilitation, vol. 17, no. 1, p. 132, 2020.

[6] M. Rahmani and M. H. Rahman, "Adaptive neural network fast fractional sliding mode control of a 7-DOF exoskeleton robot," International Journal of Control, Automation and Systems, vol. 18, no. 1, pp. 124-133, 2020.

[7] F. Xiao, Y. Gao, Y. Wang, Y. Zhu, and J. Zhao, "Design and evaluation of a 7-DOF cable-driven upper limb exoskeleton," Journal of Mechanical Science and Technology, vol. 32, no. 2, pp. 855-864, 2018.

[8] O. Baser, H. Kizilhan, and E. Kilic, "Employing variable impedance (stiffness/damping) hybrid actuators on lower limb exoskeleton robots for stable and safe walking trajectory tracking," Journal of Mechanical Science and Technology, vol. 34, no. 6, pp. 2597-2607, 2020.

[9] B. J. Kruif1, E. Schmidhauser, K. S. Stadler, and L. O. Sullivan, "Simulation architecture for modelling interaction between user and elbow-articulated exoskeleton," Journal of Bionics Engineering, vol. 14, pp. 706-715, 2017.

[10] S. Kittisares, H. Nabae, G. Endo, K. Suzumori, and R. Sakurai, "Design of knee support device based on four-bar linkage and hydraulic artificial muscle," Robomech Journal, vol. 7, no. 1, p. 16, 2020.

[11] M. Ashmi, M. Anila, and K. S. Sivanandan, "Comparison of SMC and PID controllers for pneumatically powered knee orthosis," Journal of Control, Automation and Electrical Systems, vol. 32, no. 5, pp. 1153-1163, 2021.

[12] H. D. Lee, H. Park, B. Seongho, and T. H. Kang, "Development of a soft exosuit system for walking assistance during stair ascent and descent," International Journal of Control, Automation and Systems, vol. 18, no. 10, pp. 2678-2686, 2020.

[13] G. M. Bryan, P. W. Franks, S. Song et al., "Optimized hipknee-ankle exoskeleton assistance reduces the metabolic cost of walking with worn loads," Journal of NeuroEngineering and Rehabilitation, vol. 18, no. 1, p. 161, 2021.

[14] Z. Yang, S. Guo, Y. Liu, H. Hirata, and T. Tamiya, "An intention-based online bilateral training system for upper limb motor rehabilitation," Microsystem Technologies, vol. 27, no. 1, pp. 211-222, 2021.

[15] S. X. Deng, J. Wang, and D. C. Qiao, Sport Physiology, Higher Education Press, Beijing, China, 3rd edition, 2015.

[16] X. Cui, Signal Analysis and Processing, China Power Press, Beijing, China, 3rd edition, 2016.

[17] D. F. Zhang, MATLAB Numerical Method, China Machine Press, Beijing, China, 2010.

[18] P. Li and H. Chen, "Evaluation of green building suppliers based on IVPLTS-CBR decision-making method," International Journal of Intelligent Computing and Cybernetics, vol. 15, 2021.

[19] V. C. Maduekwe and S. A. Oke, "Novel Taguchi scheme-based DEMATEL methods and DEMATEL method for the principal performance indicators of maintenance in a food processing industry," International Journal of Intelligent Computing and Cybernetics, vol. 14, no. 3, pp. 363-397, 2021.

[20] M. Lin, Z. Chen, Z. Xu, X. Gou, and F. Herrera, "Score function based on concentration degree for probabilistic linguistic term sets: an application to TOPSIS and VIKOR," Information Sciences, vol. 551, pp. 270-290, 2021.
[21] M. Lin, C. Huang, Z. Xu, and R. Chen, "Evaluating IoT platforms using integrated probabilistic linguistic MCDM method," IEEE Internet of Things Journal, vol. 7, no. 11, pp. 11195-11208, 2020.

[22] M. Lin, Y. Chen, and R. Chen, "Bibliometric analysis on Pythagorean fuzzy sets during 2013-2020," International Journal of Intelligent Computing and Cybernetics, vol. 14, no. 2, pp. 104-121, 2021.

[23] X. Q. Xu, J. L. Xie, N. Yue, and H. Wang, "Probabilistic uncertain linguistic TODIM method based on the generalized Choquet integral and its application," International Journal of Intelligent Computing and Cybernetics, vol. 14, pp. 122-144, 2021.

[24] Y. Chen, G. L. Tao, and H. Liu, "High precision adaptive robust neural network control of a servo pneumatic system," Applied Sciences, vol. 9, no. 17, p. 3472, 2019.

[25] F. L. Jiang, H. Liu, and D. X. Chai, "Humanoid lower limb: design, analysis, observer-based fuzzy adaptive control and experiment," Mathematical Problems in Engineering, vol. 2021, 15 pages, 2021. 